\title{
Preliminary Clinical Study Using a Novel Wound Dressing Composed of Hyaluronic Acid and Collagen Containing EGF
}

\author{
Akane Yu',2, Akira Takeda², Kenichi Kumazawa ${ }^{3}$, Hiroshi Miyoshi', Misato Kuroyanagi2, \\ Toshihiro Yoshitake², Eiju Uchinuma², Ryusuke Suzuki1, Yoshimitsu Kuroyanagi1* \\ ${ }^{1}$ R\&D Center for Artificial Skin, Kitasato University, Kanagawa, Japan \\ ${ }^{2}$ Department of Plastic and Aesthetic Surgery, Kitasato University, Kanagawa, Japan \\ ${ }^{3}$ Kitasato University Hospital Emergency Center, Kanagawa, Japan \\ ${ }^{4}$ Sagamihara Kyodo Hospital, Kanagawa, Japan \\ Email: ${ }^{*}$ kuroyana@ahs.kitasato-u.ac.jp
}

Received 10 February 2015; accepted 23 March 2015; published 25 March 2015

Copyright (C) 2015 by authors and Scientific Research Publishing Inc.

This work is licensed under the Creative Commons Attribution International License (CC BY). http://creativecommons.org/licenses/by/4.0/

(c) (i) Open Access

\section{Abstract}

This clinical trial aimed to evaluate the efficacy and safety of a novel wound dressing composed of hyaluronic acid (HA) and collagen (Col) containing epidermal growth factor (EGF), referred to as EGF-wound dressing. EGF-wound dressing was prepared by freeze-drying a mixed aqueous solution of high-molecular-weight HA, low-molecular-weight HA and heat-denatured Col containing EGF. EGF-wound dressing was applied to skin defects, such as intractable skin ulcers, burn ulcers, traumatic skin defects and skin donor-site wounds. The dressing was changed twice a week for a period of 6 weeks or longer, if necessary. The primary endpoints were size of wound area, formation of granulation tissue, extent of epithelialization, infection control and macroscopic appearance. Effectiveness, safety and overall clinical evaluation were scored by plastic surgeons, as authorized by the Japanese Society of Plastic and Reconstructive Surgery. This study was registered with the University Hospital Medical Information Network (UMIN0000005264). Healthy granulation tissue and rapid epithelialization were observed for a given period after application of EGFwound dressing onto the wounds. Most cases were assessed as having achieved good or excellent results. This clinical study demonstrated that EGF-wound dressing was beneficial in the treatment of various skin defects.

\section{Keywords}

Hyaluronic Acid, Collagen, Epidermal Growth Factor, Wound Dressing

\footnotetext{
${ }^{*}$ Corresponding author.
}

How to cite this paper: Yu, A., Takeda, A., Kumazawa, K., Miyoshi, H., Kuroyanagi, M., Yoshitake, T., Uchinuma, E., Suzuki, R. and Kuroyanagi, Y. (2015) Preliminary Clinical Study Using a Novel Wound Dressing Composed of Hyaluronic Acid and Collagen Containing EGF. Open Journal of Regenerative Medicine, 4, 6-13. http://dx.doi.org/10.4236/ojrm.2015.41002 


\section{Introduction}

Growth factors are known to accelerate wound healing by regulating various cell functions such as proliferation, differentiation, migration, morphogenesis and apoptosis [1] [2]. Currently, several growth factor products are commercially available. Prior to clinical use, these products must be dissolved in the accompanying solution, preserved in a refrigerator and used within a specific time period. When aqueous solutions containing the growth factor are sprayed onto a wound site, a top dressing needs to be placed on the wound as a covering material. In addition, growth factor needs to be applied daily, as free growth factor has a short half-life at the wound site.

In order to overcome these disadvantages, we have developed a novel wound dressing composed of HA and Col containing EGF (EGF-wound dressing) [3]-[7]. EGF-wound dressing was designed to contain 1.0 $\mu \mathrm{g}$ EGF per $1 \mathrm{~cm}^{2}$ of spongy sheet based on the results in a culture system [4]. We previously reported that EGF-wound dressing stimulated fibroblasts to produce increased amounts of vascular endothelial growth factor (VEGF) and hepatocyte growth factor (HGF) that were essential for angiogenesis. In practice, cytokine production of fibroblasts was assessed in a wound surface model using a fibroblast-incorporating Col gel sheet (cultured dermal substitute; CDS). CDS was elevated to the air-medium interface, on which a wound dressing was placed and cultured for 7 days. Fibroblasts in CDS covered with EGF-wound dressing released 3.6 times more VEGF and 4.6 times more HGF when compared with the wound dressing without EGF [6]. These findings are useful to support the clinical study. Animal experiments using Sprague-Dawley rats and diabetic mice demonstrated that EGF-wound dressing promoted successful wound healing associated with enhanced epithelialization and wellvascularized granulation tissue formation. In this preliminary clinical study, we evaluated the efficacy and safety of EGF-wound dressing in the treatment of various skin defects.

\section{Materials and Methods}

\subsection{Preparation of EGF-Wound Dressing}

The preparation procedure for EGF-wound dressing was as described in our previous article [5] and is briefly mentioned here. EGF-wound dressing was prepared by freeze-drying a mixed aqueous solution of high-molecular-weight HA, low-molecular-weight HA and heat-denatured Col containing EGF. Both sides of the spongy sheet were exposed to UV irradiation to induce cross-linkage between Col molecules, followed by sterilization at $110^{\circ} \mathrm{C}$ for $1 \mathrm{~h}$. The resulting spongy sheet is about $5 \mathrm{~mm}$ in thickness, containing EGF at a concentration of 1 $\mu \mathrm{g}$ per $1 \mathrm{~cm}^{2}$ of spongy sheet.

\subsection{Overview of Clinical Trial}

The protocol for this study and any amendments were reviewed and approved by Kitasato University Hospital Ethics Committee in April 2011 (C10-656). This study was conducted in accordance with the Declaration of Helsinki, and the ethical guidelines for clinical studies of the Ministry of Health, Labour and Welfare of Japan, and was registered with the University Hospital Medical Information Network (UMIN0000005264). This study was conducted at Kitasato University Hospital and JA Kanagawa Sagamihara Kyodo Hospital.

All participants provided written, informed consent before any study-specific procedures were undertaken. Inclusion criteria were: 1) patients aged 16 years or older at the time of providing informed consent; 2) patients with intractable skin ulcers not healing with conventional treatments for more than one month;3) patients with burn injuries, donor-site wounds or traumatic skin defects; and 4) patients providing written informed consent. Exclusion criteria were: 1) history of allergy to drugs or dressings to be used in the trial; 2) past history of any malignant tumor at the wound site; and 3) judged by the investigator or sub-investigator to be inappropriate as a subject of this study. There are no potential conflicts of interest to disclose.

\subsection{Application of EGF-Wound Dressing}

Wound surfaces were debrided as necessary and rinsed with physiological saline solution. EGF-wound dressings, cut according to the shape of the wound, were then applied to the wound site, on which a commercially available polyurethane film dressing was used as a top dressing. In the case of wounds associated with excess amounts of exudate, conventional ointment-gauze dressing was used as a top dressing. EGF-wound dressings were applied repeatedly at intervals of three to five days for a period of six weeks, or longer if necessary. 


\subsection{Wound Assessments}

Every time the dressings were changed, the size of the wound was measured and digital images of the wounds were taken with a ruler placed on the skin adjacent to the wounds using a digital camera for macroscopic assessment.

Primary endpoints were effectiveness, safety and overall clinical evaluation, as scored by authorized plastic surgeons. Formation of granulation tissue, extent of epithelialization, control of infection and management of exudate were evaluated according to the protocol. Table 1 shows the effectiveness score (total 0 - 100 pts). Table 2 shows the safety evaluation. Table 3 shows the overall clinical evaluation. Based on the scores, wound conditions were evaluated for safety as very safe, mostly safe, problematically safe or not safe, and for clinical evaluation as excellent, good, fair or poor. Patient satisfaction was evaluated as a secondary endpoint. At the end of each clinical trial, patients themselves scored their satisfaction for the treatment with a $10 \mathrm{~cm}$ visual analogue scale (VAS): a score of 0 indicated the greatest dissatisfaction, and a 10 indicated the greatest satisfaction.

\section{Results}

\subsection{Outcome of Clinical Study}

For the present 16 clinical cases (male 7, female 9), mean age was $59 \pm 20$ years (mean \pm SD). Table 4 shows the indications and outcomes of the clinical study. Lower limb lesions were seen in $90 \%$ of all cases. For outcome, $93.7 \%$ (15/16) of various wounds were evaluated as achieving good or excellent results, including $100 \%$ (3/3) of DB and DDB, $100 \%$ (2/2) of partial-thickness donor wounds, $100 \%(2 / 2)$ of traumatic skin defects, $88.9 \%$ (8/9) of intractable skin ulcers. For safety, $87.5 \%$ (14/16) were evaluated as very safe without any problems, and $12.5 \%(2 / 16)$ showed mostly safe with minor problems that could be treated immediately. Two patients exhibited light contact dermatitis, but were able to continue with the investigation after appropriate treatment. No patient suffered from infections.

Before application of EGF-wound dressing, patient satisfaction scores for efficacy of wound healing and relief from pain were $4.7 \pm 2.3$ and $4.8 \pm 3.1$, respectively. After application, patient satisfaction scores for efficacy

Table 1. Effectiveness score (total 0 - 100 pts).

\begin{tabular}{cccccc}
\hline & Much better & Better & Equal & Worse & Much worse \\
\hline Granulation & 35 & 27 & 18 & 9 & 0 \\
Epithelialization & 35 & 27 & 18 & 9 & 0 \\
Infection & 15 & 13 & 8 & 4 & 0 \\
Exudate & 15 & 13 & 8 & 4 & 0 \\
\hline
\end{tabular}

Table 2. Safety evaluation.

\begin{tabular}{cc}
\hline A & Very safe \\
B & Mostly safe \\
C & Problematically safe \\
D & Not safe (infection) \\
\hline
\end{tabular}

Table 3. Overall clinical evaluation.

\begin{tabular}{cc} 
& Total effective score and safety assessment \\
\hline Excellent & $80-100$ \& A \\
Good & $60-79$ \& A or B \\
Fair & $40-59 \&$ A or B or C, or $>60$ \& C \\
Poor & $<40$ \& any A-D, or $>40$ \& D \\
\hline
\end{tabular}


and relief from pain were $8.6 \pm 2.0$ and $8.4 \pm 2.0$, respectively. The mean score for total patient satisfaction was $8.6 \pm 2.1$ (mean $\pm \mathrm{SD}$ ) (Table 5).

\subsection{Representative Cases}

Case 1: A 64-year-old-woman with chronic renal failure developed a skin ulcer on the left leg. One month after receiving ointment therapy, the wound appeared as a hollowed ulcer with $3 \times 1.8 \mathrm{~cm}$ in size (Figure 1(a)). After application of EGF-wound dressings twice a week on an outpatient basis, good granulation tissue formed and epithelialization was observed from the edge of the wound after 2 weeks (Figure 1(b)). Wound size was significantly smaller at 1 month (Figure 1(c)), and wound closure was achieved in 2 months (Figure 1(d)).

Table 4. Indication and outcome of clinical study.

\begin{tabular}{cccccc}
\hline Indication & Excellent & Good & Fair & Poor & Total \\
\hline DDB/DB $^{*}$ & 2 & 1 & 0 & 0 & 3 \\
Donor wounds & 0 & 2 & 0 & 0 & 2 \\
Traumatic wounds & 1 & 1 & 0 & 0 & 2 \\
Intractable skin ulcers & 5 & 3 & 0 & 1 \\
\hline
\end{tabular}

"DDB: deep dermal burn (second burn). DB: dermal burn (third burn).

Table 5. Patient satisfaction score (Wilcoxon signed rank test. ${ }^{*} \mathrm{p}<0.05$ ).

\begin{tabular}{ccc} 
& Before EGF-dressing & After EGF-dressing \\
\hline Efficacy of treatment & $4.7 \pm 2.3$ & $8.6 \pm 2.0^{*}$ \\
Relief from pain & $4.8 \pm 3.1$ & $8.4 \pm 2.0^{*}$ \\
\hline
\end{tabular}
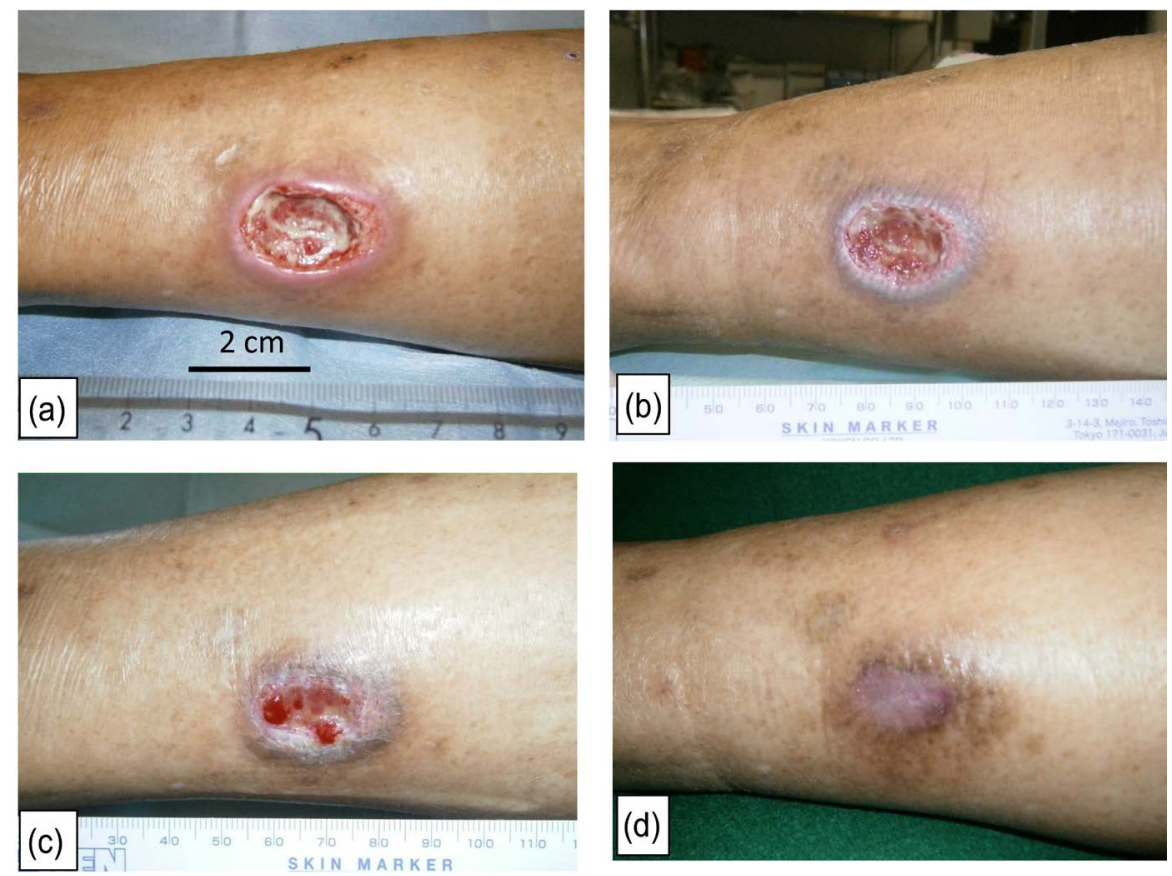

Figure 1. A 64-year-old-woman with chronic renal failure developed a skin ulcer on the left leg. One month after receiving ointment therapy, the wound appeared as a hollowed ulcer with $3 \times 1.8 \mathrm{~cm}$ in size (a). After application of EGF-wound dressings twice a week on an outpatient basis, good granulation tissue formed and epithelialization was observed from the edge of the wound after 2 weeks (b). Wound size was significantly smaller at 4 weeks (c), and wound closure was achieved at 2 months (d). Effectiveness: 100, Safety: A, overall evaluation: excellent. 
Case 2: A 65-year-old woman with severe heart failure developed a skin ulcer on the right knee $(5.3 \times 4.8 \mathrm{~cm})$. After recovering from heart surgery, the wound was debrided (Figure 2(a)) and EGF-wound dressings were applied twice a week (Figure 2(b)). Wound condition improved significantly and good granulation tissue and well epithelialization was observed at 2 months (Figure 2(c)). Full-thickness auto-skin graft was performed at this time. Unpleasant contraction was not observed at 2 months after surgery (Figure 2(d)).

Case 3: A 60-year-old man developed a skin ulcer on the left ankle. Although he had received conventional therapy for 2 months, the wound was in poor condition with excessive exudate $(3.5 \times 1.7 \mathrm{~cm})$ (Figure 3(a)). EGF dressing was applied to the site every twice a week (Figure 3(b)). The wound conditions improved at an early stage and epithelialization from the outer margins was observed $(2.0 \times 0.7 \mathrm{~cm})$ at 2 weeks (Figure 3(c)). Wound closure was achieved at 1 month (Figure 3(d)).

Case 4: A 27-year-old man sustained a burn ulcer on the right foot. While he had received ointment therapy for 3 weeks, the wound condition did not improve $(2.8 \times 1.5 \mathrm{~cm})$ (Figure 4(a)). After application of EGF dressing on the wound site, the wound condition improved and wound size was reduced significantly in 1 week (Figure 4(b)). Simple closure by suturing was performed at 2 weeks after application of EGF dressing (Figure 4(c)). Unpleasant contraction was not observed through monitoring the wound up to 1 month (Figure 4(d)).

Case 5: A 74-year-old woman who was complicated by rheumatoid arthritis and deep venous thrombosis, developed a traumatic ulcer on the left knee. After conventional therapy for 1 month, she still had an ulcer due to a poor condition of her underlying disease $(11 \times 3.5 \mathrm{~cm})($ Figure 5(a)). EGF dressing was applied to the wound site every twice a week (Figure 5(b)). Wound size decreased significantly with good granulation tissue and epithelialization at 6 weeks $(6.6 \times 1.8 \mathrm{~cm})($ Figure $5(\mathrm{c}))$ and 3 months $(3.1 \times 0.9 \mathrm{~cm})($ Figure $5(\mathrm{~d}))$.

\section{Discussion}

We developed a wound dressing composed of HA and Col spongy sheet containing EGF i.e., EGF-wound dressing. HA is an excellent biomaterial for wound healing because of its unique hygroscopic, rheological and viscoelastic properties [8] [9]. High-molecular-weight HA creates an excellent wound healing environment, while low-molecular-weight HA induces angiogenesis [10]-[12]. Col and Col-derived peptides act as a chemoattractant for fibroblasts in vitro and may have a similar activity in vivo [13]. We previously reported the efficacy
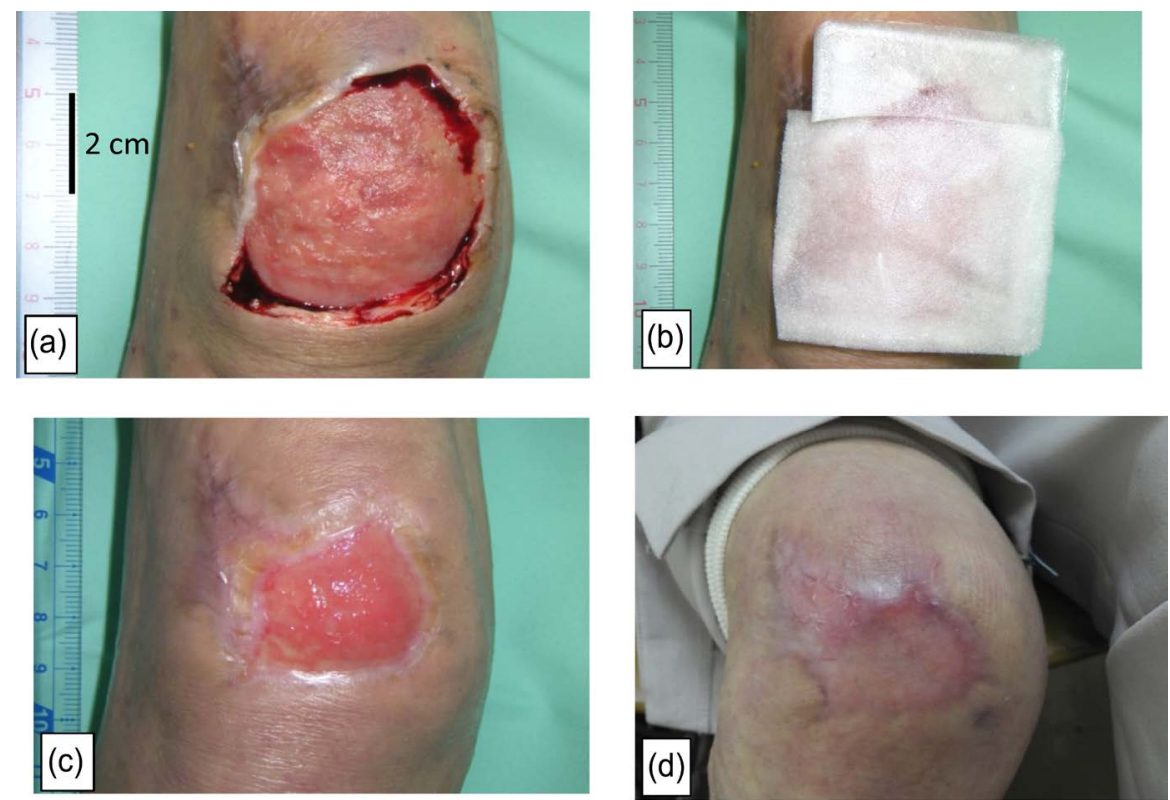

Figure 2. A 65-year-old woman with severe heart failure developed a skin ulcer on the right knee $(5.3 \times 4.8 \mathrm{~cm})$. After recovering from heart surgery, the wound was debrided (a) and EGF-wound dressings were applied twice a week (b). Wound condition improved significantly and good granulation tissue and well epithelialization was observed. Full-thickness auto-skin graft was performed at 8 weeks (c). Unpleasant contraction was not observed at 2 months after surgery (d). Effectiveness: 88, Safety: A, overall evaluation: excellent. 

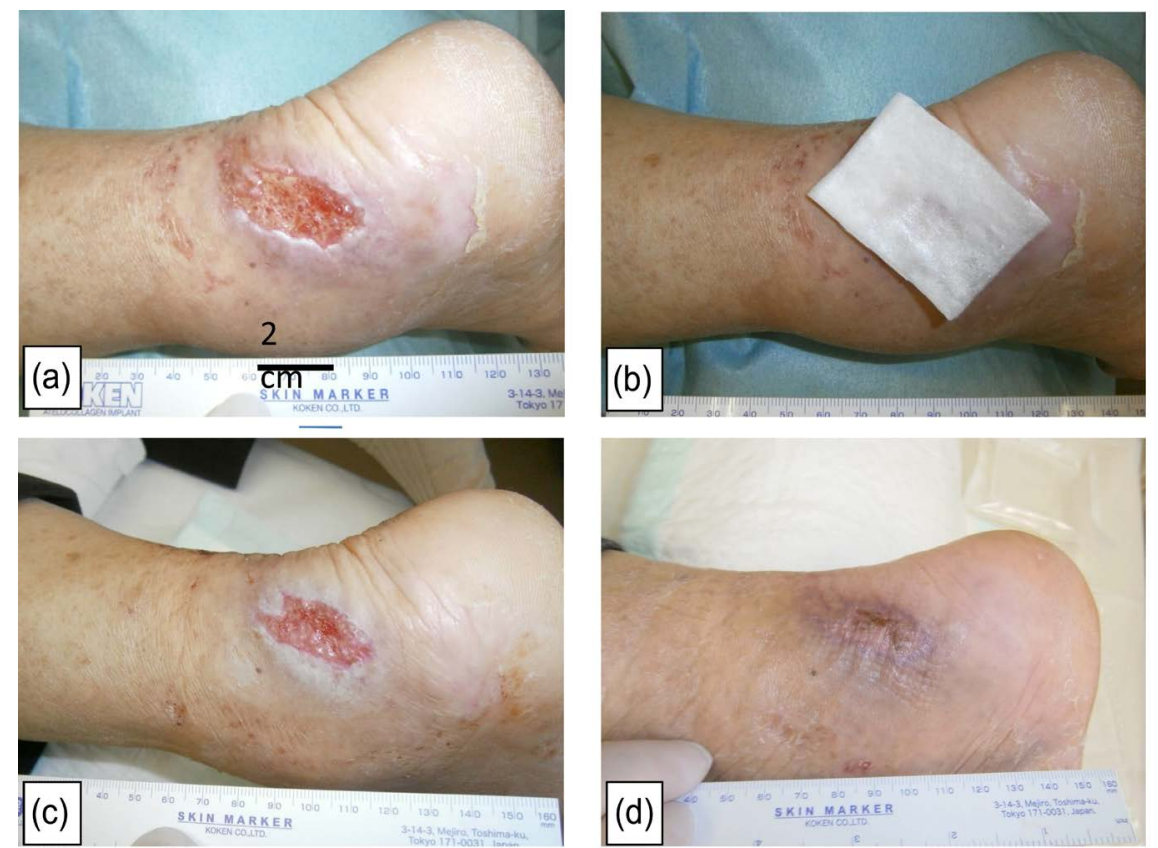

Figure 3. A 60-year-old man developed a skin ulcer on the left ankle. Although he had received conventional therapy for 2 months, the wound was in poor condition with excessive exudate $(3.5 \times 1.7 \mathrm{~cm})(\mathrm{a})$. EGF dressing was applied to the site every twice a week (b). The wound conditions improved at an early stage and epithelialization from the outer margins was observed $(2.0 \times 0.7 \mathrm{~cm})$ at 2 weeks $(\mathrm{c})$. Wound closure was achieved in 4 weeks (d). Effectiveness: 90, Safety: A, overall evaluation: excellent.
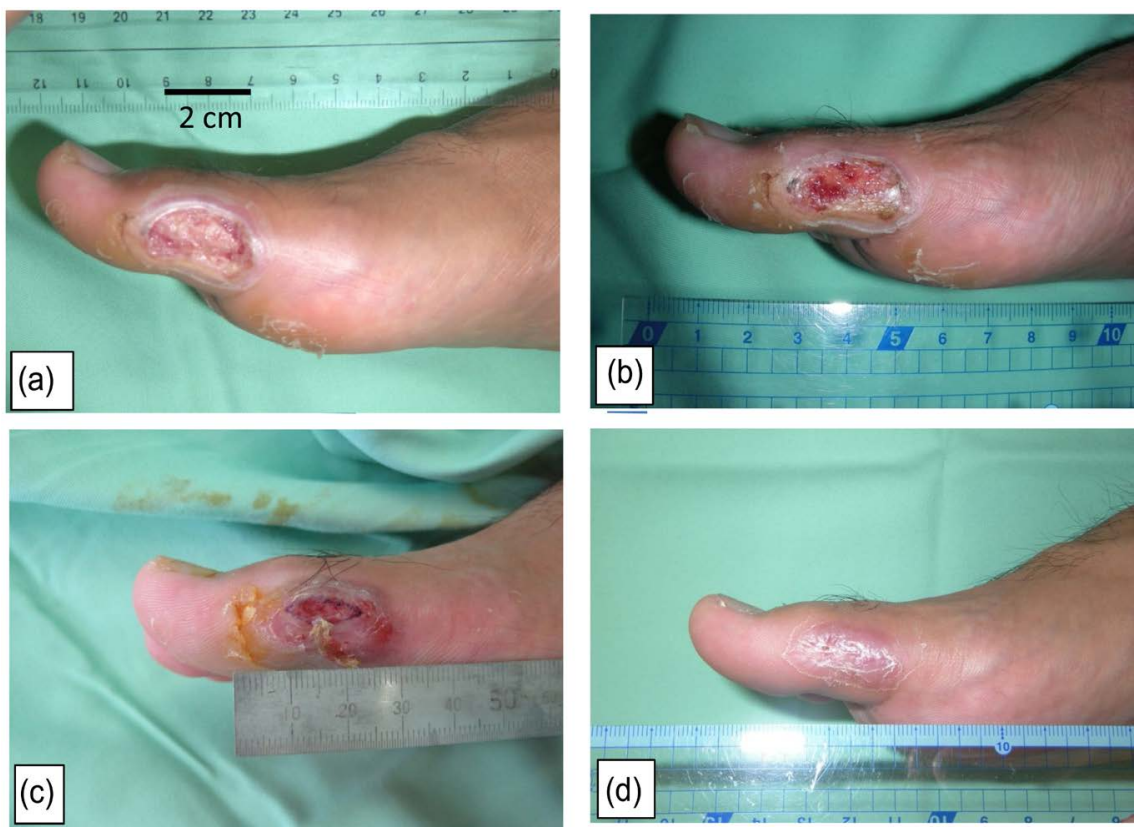

Figure 4. A 27-year-old man sustained a burn ulcer on the right foot. While he had received ointment therapy for 3 weeks, the wound condition did not improve $(2.8 \times 1.5 \mathrm{~cm})$ (a). After application of EGF dressing on the wound site, the wound condition improved and wound size was reduced significantly in one week (b). Simple closure by suturing was performed at 2 weeks after application of EGF dressing (c). Unpleasant contraction was not observed through monitoring the wound up to 1 month (d). Effectiveness: 91, Safety: A, overall evaluation: excellent. 

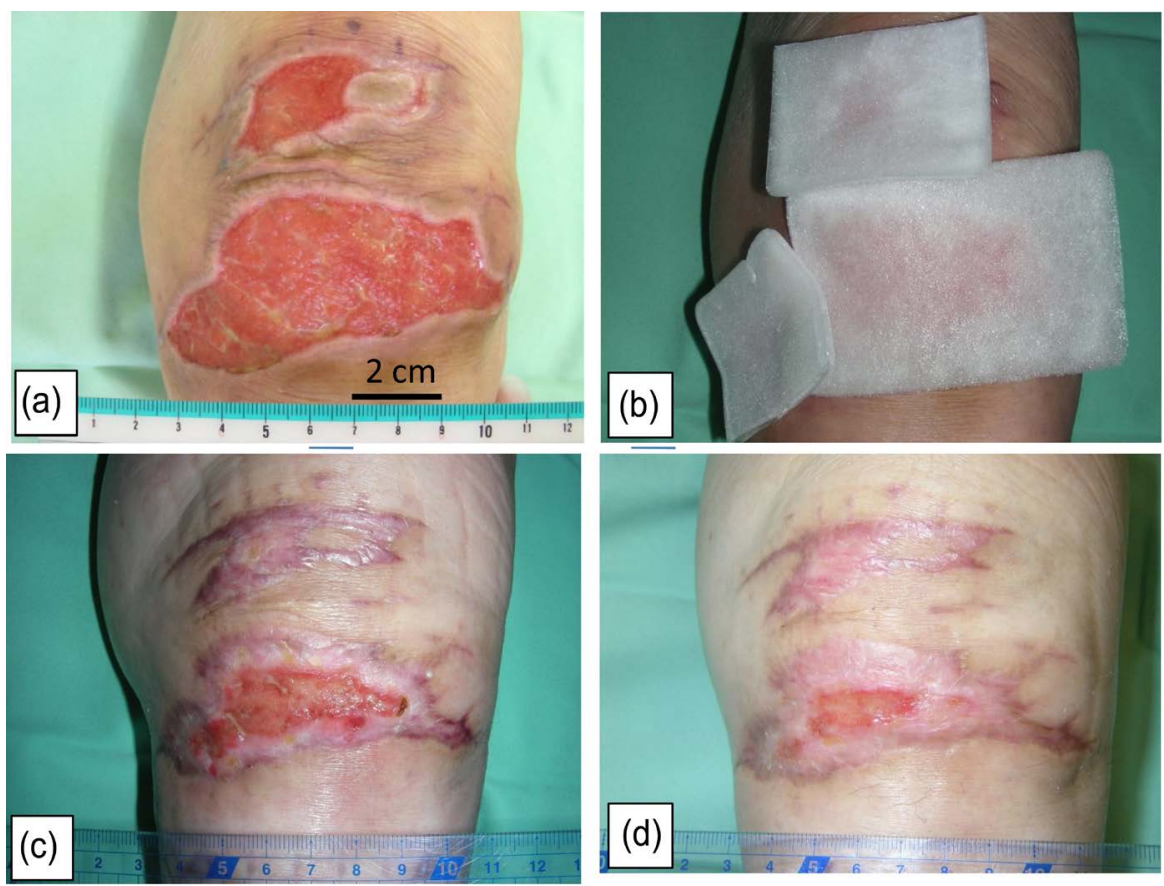

Figure 5. A 74-year-old woman who was complicated by rheumatoid arthritis and deep venous thrombosis, developed a traumatic ulcer on the left knee. After 1 month conventional therapy, she still had an ulcer due to a poor condition of her underlying disease $(11 \times$ $3.5 \mathrm{~cm}$ ) (a). EGF dressing was applied to the wound site every twice a week (b). Wound size decreased significantly with good granulation tissue and epithelialization at 6 weeks $(6.6 \times 1.8 \mathrm{~cm})(\mathrm{c})$ and at 3 months $(3.1 \times 0.9 \mathrm{~cm})(\mathrm{d})$. Effectiveness: 88 , Safety: A, overall evaluation: excellent.

of EGF-wound dressing for the production of angiogenic growth factor in culture systems [4] [6] and for the enhancement of granulation tissue formation associated with epithelialization in animal tests using rats and diabetic mice [3] [5]. Based on our previous studies, highly hydrated HA molecules apparently allow EGF released from the wound dressing to remain stable for enough time to initiate cell proliferation and cytokine production in vitro. Based on animal studies, we suspect that re-epithelialization is facilitated by the direct action of EGF released from wound dressing and that angiogenesis is facilitated indirectly by increased VEGF and HGF production by fibroblasts in the granulation tissue. These actions need to be confirmed in a clinical study.

Most clinical cases were assessed as having achieved good or excellent results without critical problems. These data suggest that EGF-wound dressing can provide safe and effective therapy for patients with various types of problematic wound, such as burns, chronic ulcers and traumatic skin defects. From the viewpoint of patient satisfaction, scores for efficacy and relief from pain rose significantly, when compared with the state before using EGF-wound dressing. After application of EGF-wound dressing, most patients were likely to feel better and more satisfied with this treatment.

In all clinical cases, EGF-wound dressing was applied repeatedly at intervals of three to five days for a period of six weeks, or longer if necessary. In the case of commercially available growth factor products, aqueous solutions of growth factor need to be sprayed on a wound site daily, because of their rapid inactivation after topical administration. In addition, the top dressing must be changed daily. However, the present EGF-wound dressing can overcome the disadvantages associated with commercially available growth factor products.

\section{References}

[1] Singer, A.J. and Clark, R.A. (1999) Cutaneous Wound Healing. New England Journal of Medicine, 341, 738-746. http://dx.doi.org/10.1056/NEJM199909023411006

[2] Moulin, V. (1995) Growth Factors in Skin Wound Healing. European Journal of Cell Biology, 68, 1-7.

[3] Kondo, S. and Kuroyanagi, Y. (2012) Development of a Wound Dressing Composed of Hyaluronic Acid and Collagen 
Sponge with Epidermal Growth Factor. Journal of Biomaterials Science, Polymer Edition, 23, 629-643. http://dx.doi.org/10.1163/092050611X555687

[4] Yu, A., Matsuda, Y., Takeda, A., Uchinuma, E. and Kuroyanagi, Y. (2012) Effect of EGF and bFGF on Fibroblast Proliferation and Angiogenic Cytokine Production from Cultured Dermal Substitutes. Journal of Biomaterials Science, Polymer Edition, 23, 1315-1324.

[5] Kondo, S., Niiyama, H., Yu, A. and Kuroyanagi, Y. (2012) Evaluation of a Wound Dressing Composed of Hyaluronic Acid and Collagen Sponge Containing Epidermal Growth Factor in Diabetic Mice. Journal of Biomaterials Science, Polymer Edition, 23, 1729-1740. http://dx.doi.org/10.1163/092050611X555687

[6] Yu, A., Niiyama, H., Kondo, S., Yamamoto, A., Suzuki, R. and Kuroyanagi, Y. (2013) Wound Dressing Composed of Hyaluronic Acid and Collagen Containing EGF or bFGF: Comparative Culture Study. Journal of Biomaterials Science, Polymer Edition, 24, 1015-1026. http://dx.doi.org/10.1080/09205063.2012.731375

[7] Niiyama, H. and Kuroyanagi, Y. (2014) Development of Novel Wound Dressing Composed of Hyaluronic Acid and Collagen Sponge Containing Epidermal Growth Factor and Vitamin C Derivative. Journal of Artificial Organs, 17, 8187. http://dx.doi.org/10.1007/s10047-013-0737-x

[8] Chen, W.Y. and Abatangelo, G. (1999) Functions of Hyaluronan in Wound Repair. Wound Repair and Regeneration, 7, 79-89. http://dx.doi.org/10.1046/j.1524-475X.1999.00079.x

[9] Laurent, T.C. and Fraser, J.R. (1992) Hyaluronan. FASEB Journal, 6, 2397-2404.

[10] West, D.C., Hampson, I.N., Arnold, F. and Kumar, S. (1985) Angiogenesis Induced by Degradation Products of Hyaluronic Acid. Science, 228, 1324-1326. http://dx.doi.org/10.1126/science.2408340

[11] Sattar, A., Rooney, P., Kumar, S., Pye, D., West, D.C., Scott, I. and Ledger, P. (1994) Application of Angiogenic Oligosaccharides of Hyaluronan Increases Blood Vessel Numbers in Rat Skin. Journal of Investigative Dermatology, 103, 576-579. http://dx.doi.org/10.1111/1523-1747.ep12396880

[12] Lees, V.C., Fan, T.P. and West, D.C. (1995) Angiogenesis in a Delayed Revascularization Model Is Accelerated by Angiogenic Oligosaccharides of Hyaluronan. Laboratory Investigation, 73, 259-266.

[13] Postlethwaite, A.E., Seyer, J.M. and Kang, A.H. (1978) Chemotactic Attraction of Human Fibroblasts to Type I, II, and III Collagens and Collagen-Derived Peptides. Proceedings of the National Academy of Sciences of the United States of America, 75, 871-875. http://dx.doi.org/10.1073/pnas.75.2.871 